\title{
Migration du calcium et des phosphates inorganiques dans les fromages à pâte molle de type Camembert au cours de l'affinage
}

\author{
par \\ Y. LE GRAET, A. LEPIENNE, G. BRULE \\ et P. DUCRUET*
}

R és u m é

Au cours de l'affinage des fromages à pâte molle de type camembert, le calcium et les phosphates migrent de l'intérieur vers la croûte où les teneurs en ces deux éléments peuvent respectivement dépasser 17 et $9 \mathrm{~g} / \mathrm{kg}$. La migration de ces minéraux coïncide avec le développement du Penicillium caseicolum mais se trouve en réalité sous la dépendance du $\mathrm{pH}$; en effet, c'est par suite du développement de Penicillium caseicolum que se crée un gradient de $\mathrm{pH}$ dans le fromage. Des fromages qui ont reçu une pulvérisation d'une solution antifongique, mais qui se trouvent en atmosphère ammoniacale $(\mathrm{pH} 9,5)$ avec par conséquent un gradient de $\mathrm{pH}$, d'origine chimique et non biologique subissent une migration minérale analogue à celle observée dans les fromages avec Penicillium.

Des modifications des teneurs en calcium et en phosphates des fromages ont permis de montrer que les phosphates sont le facteur limitant de la migration; les teneurs finales en phosphore d'un fromage se situent aux environs de $2 \mathrm{~g} / \mathrm{kg}$ ce qui correspond au phosphore organique. L'addition de phosphate disodique aux sels de saumure permet une migration plus importante du calcium.

Mots clés :

Affinage - Calcium - Camembert - Minéraux - Phosphate - Ammoniac.

\footnotetext{
* Institut National de la Recherche Agronomique, Laboratoire de Recherches de
} Technologie Laitière, 65, rue de Saint-Brieuc - 35042 Rennes cedex (France). 


\section{S u m m a ry}

\section{MINERAL MIGRATION IN SOFT CHEESES DURING RIPENING}

During ripening of soft cheeses, calcium and phosphorus are migrating from inside to outside. Amounts of these elements at outside are respectively upper to 17 and $9 \mathrm{~g} / \mathrm{kg}$. Mineral migration is starting with Penicillium caseicolum growth; it is dependent on the evolution of $\mathrm{pH}$. This migration is observed in cheeses treated with pimaricine and placed in ammoniacal atmosphere.

By modifying initial amounts of calcium and phosphorus either by ultrafiltration of milk before cheesemaking or by addition of these elements in brine inside, phosphorus amounts of cheese always decreases to $2 \mathrm{~g} / \mathrm{kg}$. This value corresponds to organic phosphorus. Totality of mineral phosphorus migrates to the cheese surface; final concentration of calcium in the cheese curd is dependent on mineral phosphorus content.

Key words:

Ripening, - Mineral - Calcium - Phosphorus - Camembert - Ammonia.

\section{INTRODUCTION}

La composition minérale d'un fromage dépend étroitement des paramètres technologiques mis en œuvre pour l'obtenir; les teneurs en calcium et phosphate ainsi que le rapport de ces deux éléments permettent de caractériser un très grand nombre de variétés (Guittonneau et Chevalier, 1935; Randoin et Jourdan, 1951; Gueguen, 1979). Les taux de calcium, magnésium et phosphates inorganiques associés aux protéines et peptides déterminent, pour une part importante, les caractéristiques rhéologiques du fromage. Ils évoluent au cours de l'affinage étant donné qu'ils dépendent principalement $\mathrm{du} \mathrm{pH}$. Suivant que la protéolyse et la dégradation de l'acide lactique s'effectuent dans la masse du fromage ou à sa surface, le gradient de $\mathrm{pH}$ entre l'intérieur et l'extérieur est plus ou moins important; dans le cas des pâtes molles de type camembert, ce gradient s'établit dès le développement du Penicillium. Metche et Fanni (1978) ont mis en évidence une migration importante du calcium et des phosphates vers l'extérieur des pâtes molles au cours de l'implantation de la moisissure de surface. Ils ont attribué cette migration au pouvoir séquestrant du Penicillium.

Nous nous sommes attachés, dans ce travail, à déterminer l'influence du $\mathrm{pH}$ de surface sur la migration minérale indépendamment de celle plus complexe du Penicillium. De plus, nous avons examiné l'incidence des teneurs initiales en calcium et phosphates du fromage, qui varient suivant les paramètres technologiques mis 
en œuvre ou qui peuvent être modifiées par introduction de l'un de ces éléments au moment du salage.

\section{MATERIEL ET METHODES}

\section{Matière première}

Les fromages à pâte molle de type camembert étaient fabriqués à partir d'un lait pasteurisé $72^{\circ} \mathrm{C}-15 \mathrm{~s}$, soit selon le procédé traditionnel, soit selon le procédé M.M.V. (Maubois et al., 1969); dans ce dernier cas le lait était ultrafiltré à la température de $50^{\circ} \mathrm{C}$ sur un module SFEC à $\mathrm{pH}$ 6,6 et concentré 5,6 fois. Les fromages obtenus avaient un rapport gras/sec de $45 \%$.

\section{Salage}

Les fromages étaient salés soit en saumure à saturation, soit à sec. Pour éviter les pertes au cours du salage à sec, chaque fromage était placé sur une feuille d'aluminium et recevait $3,8 \mathrm{~g}$ de chlorure de sodium.

Pour l'étude de l'influence de différents sels de saumure certains fromages ont reçu, à sec, en plus des $3,8 \mathrm{~g}$ de chlorure de sodium, $3 \mathrm{~g}$ de phosphate disodique $\left(\mathrm{Na}_{2} \mathrm{HPO}_{4} 2 \mathrm{H}_{2} \mathrm{O}\right)$ ou $3 \mathrm{~g}$ de chlorure de calcium $\left(\mathrm{CaCl}_{2}\right)$.

\section{Affinage}

Après salage, les fromages étaient divisés en 2 lots, l'un ayant été ensemencé par pulvérisation d'une suspension de spores de Penicillium caseicolum, l'autre ayant été traité à l'aide d'une solution antifongique de pimaricine à $0,5 \%$ (Gist-Brocades NV) afin d'éviter tout développement de Penicillium caseicolum. Enfin chacun des 2 lots obtenus, (Penicillium et pimaricine), était à nouveau divisé en 2 pour être affiné dans une enceinte ventilée d'un volume de $0,75 \mathrm{~m}^{3}$, soit en atmosphère non modifiée (12० $\mathrm{C}$ hygrométrie $95 \%$ ) (fromages témoins), soit en atmosphère d'ammoniac. La diffusion du gaz $\mathrm{NH}_{3}$ se faisait à partir d'un bécher d'un volume d'un litre contenant 0,51 d'une solution d'ammoniaque maintenue à $\mathrm{pH}$ constant $(9,5)$ à l'aide d'un pH-Stat Mettler connecté à une réserve d'ammoniaque $0,3 \mathrm{~N}$.

\section{Découpe des fromages pour analyse}

Chaque fromage était découpé en 3 parties : la croûte, la partie sous croûte, et le centre (fig. 1).

Pour les fromages dont la moisissure (Penicillium caseicolum) était bien développée la croûte était obtenue en découpant une lamelle de $7 \mathrm{~mm}$ et en enlevant soigneusement la partie sous croûte 

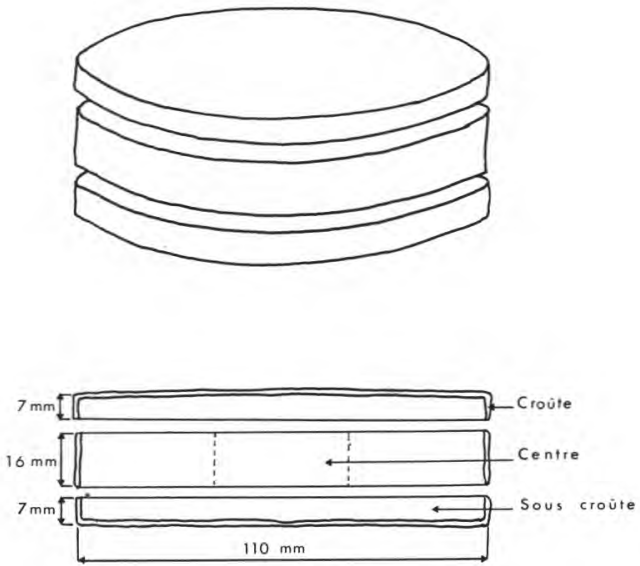

fig. 1

Découpe du fromage.

Cutting of cheese.

à la spatule, de façon à ne laisser qu'un minimum de pâte fixé à la croûte. En ce qui concerne les fromages dont le Penicillium caseicolum n'était pas, ou pas encore développé, la croûte était obtenue en découpant une lamelle aussi fine que possible $(2 \mathrm{~mm}$ environ pour tenir compte des irrégularités du fromage) à partir de la lamelle de $7 \mathrm{~mm}$ (croûte et partie sous croûte). La croûte représente environ $15 \%$ du poids d'un fromage affiné de $270 \mathrm{~g}$, la partie sous croûte $45 \%$ et le centre $40 \%$.

\section{Déterminations analytiques}

- Mesure du $\mathrm{pH}$ : les déterminations de $\mathrm{pH}$ étaient effectuées avec un appareil TACUSSEL MINISIS 5000 à 0,01 unité pH près.

- Teneur en matière sèche : elle était déterminée par dessication à l'étuve à $102-105^{\circ} \mathrm{C}$ pendant $7 \mathrm{~h}$.

- Teneur en minéraux : la détermination de la teneur en calcium était réalisée par spectrophotométrie d'absorption atomique sur un appareil Varian 1200, à partir des cendres effectuées sur le fromage et reprises dans de l'acide chlorhydrique $0,1 \mathrm{~N}$, puis suivant le protocole défini par Brulé et al. (1974). Le dosage du phosphore total était effectué selon la méthode préconisée par la Fédération Internationale de Laiterie (norme 33).

\section{RESULTATS}

\section{Répartition du calcium et du phosphore dans les fromages affinés témoins}

Nous avons déterminé les teneurs en calcium et phosphore dans le centre, la partie sous croûte et la croûte des fromages 
affinés témoins âgés de 30 jours. Les teneurs moyennes en calcium de ces 3 parties sont 0,86 pour le centre, 1,26 pour la partie souscroûte et $17,33 \mathrm{~g} / \mathrm{kg}$ pour la croûte. Les teneurs en phosphore sont 1,91 pour le centre, 1,66 pour la partie sous-croûte et $9,23 \mathrm{~g} / \mathrm{kg}$ pour la croûte (fig. 2). Il y a donc une migration du calcium et du phosphore de l'intérieur du fromage vers la croûte.

Le rapport massique $\mathrm{Ca} / \mathrm{P}$ est de 0,45 pour le centre, 0,75 pour la partie sous croûte et 1,87 pour la croûte.
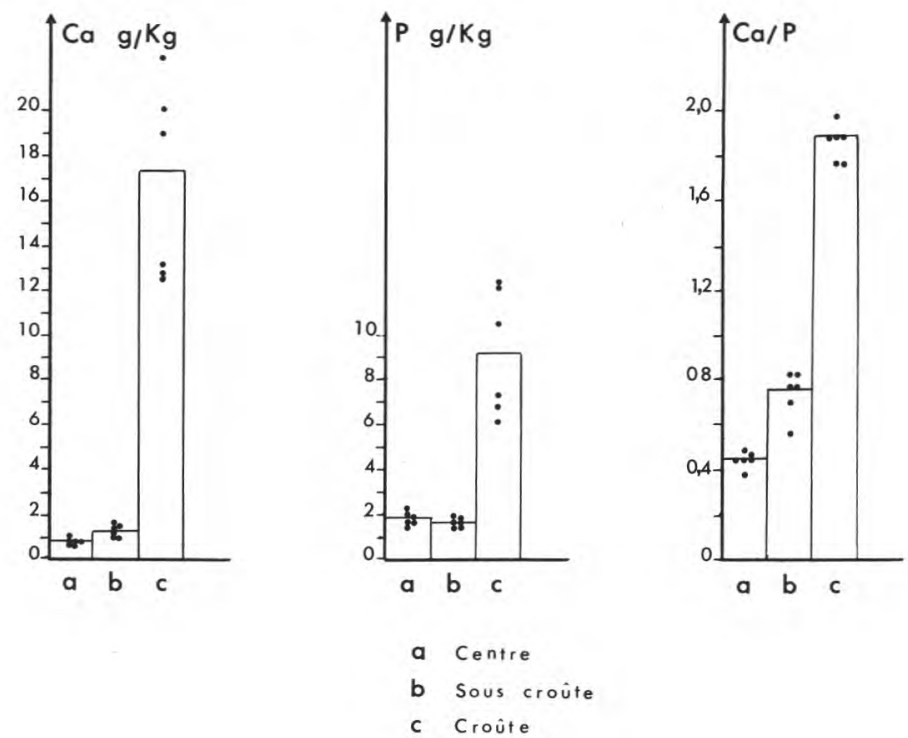

fig. 2

Répartition du calcium et phosphore dans les fromages affinés.

Calcium and phosphorus distribution in ripened cheese.

\section{Influence de l'atmosphère et du Penicillium}

a) Evolution $d u$ pH du fromage

Les variations de $\mathrm{pH}$ du fromage, couvert de Penicillium et affiné en atmosphère non modifiée, sont représentées sur la figure 3a. Le pH évolue de 4,69 à 7,20 dans la croûte, entre 3 et 9 jours, puis se stabilise vers $\mathrm{pH} 7,60$ à 17 jours. Dans la partie sous croûte le $\mathrm{pH}$ s'élève de 4,58 à 5,95 de façon linéaire à partir du $6^{\mathrm{e}}$ jour. Dans le centre l'élévation de $\mathrm{pH}$ est faible : 4,54 à 6 jours, 4,85 et 17 jours.

L'évolution des courbes de $\mathrm{pH}$ des fromages affinés en atmosphère d'ammoniac (fig. $3 \mathrm{~b}$ ) montre une remontée du $\mathrm{pH}$ plus précoce 


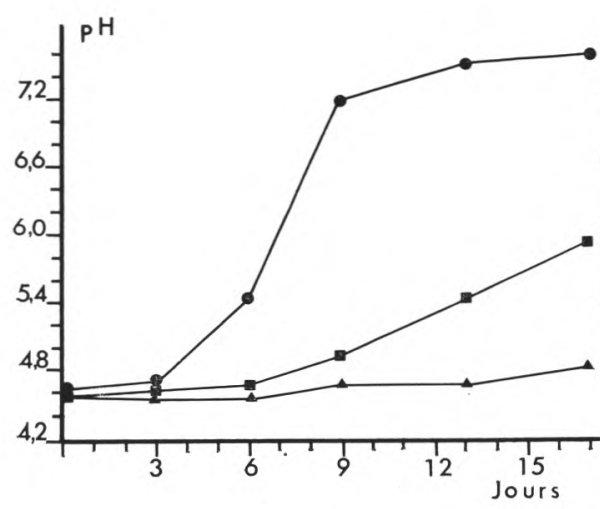

$3 a$

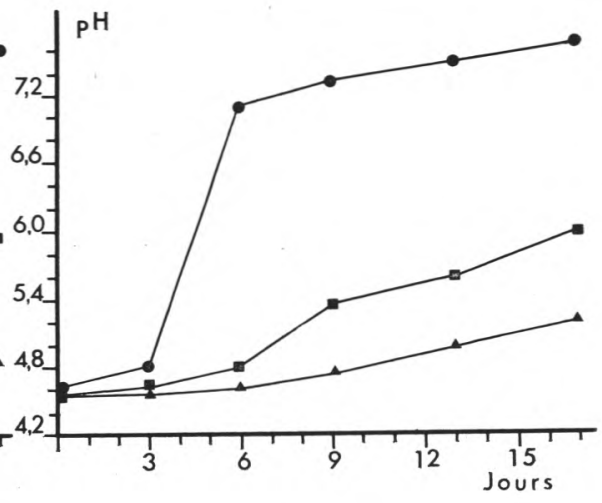

$3 \mathrm{~b}$

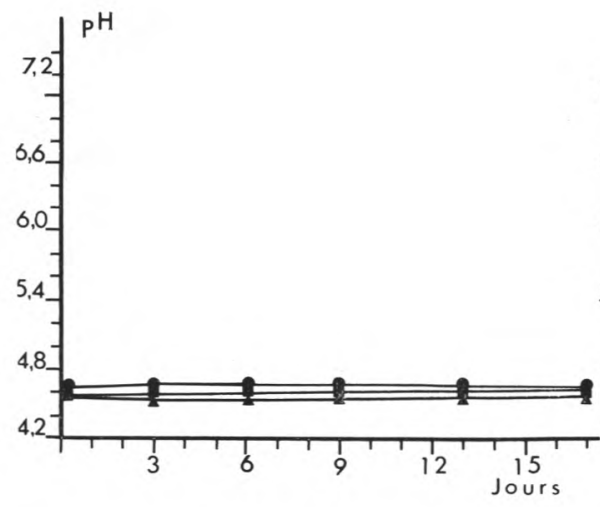

$3 c$

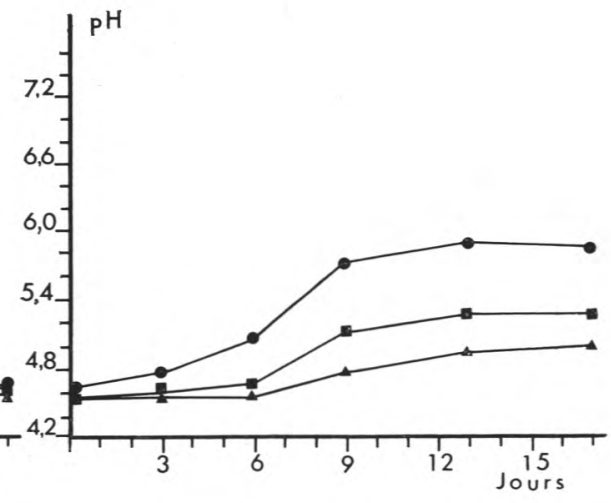

$3 d$

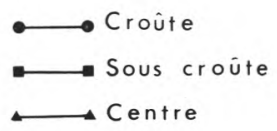

fig. 3

Evolution du $\mathrm{pH}$ au cours de l'affinage :

- fromages ensemencés en $P$. caseicolum (3a - atmosphère non modifiée ; $3 \mathrm{~b}$ - atmosphère ammoniacale) ;

- fromages traités à la pimaricine $(3 \mathrm{c}$ - atmosphère non modifiée : $3 \mathrm{~d}$ atmosphère ammoniacale pendant $13 \mathrm{j}$ ).

Evolution of $\mathrm{pH}$ during ripening:

- 3a - P. caseicolum - Normal conditions;

- $3 \mathrm{~b}$ - P. caseicolum - $\mathrm{NH}_{3}$ conditions;

- 3c - Pimaricin - Normal conditions;

- 3 d - Pimaricin - $\mathrm{NH}_{3}$ conditions. 
et plus rapide dans la croûte que dans le cas précédent. Ainsi entre 3 et 6 jours le $\mathrm{pH}$ remonte de 4,80 à 7,10. A 17 jours le $\mathrm{pH}$ atteint 7,63. Dans la partie sous croûte, l'évolution est identique à celle observée précédemment. Dans le centre le pH est de 4,55 à 3 jours et 5,20 à 17 jours. Dans le cas des fromages traités à la Pimaricine et placés en atmosphère non modifiée nous n'enregistrons aucune évolution du $\mathrm{pH}$ (fig. 3c). Par contre les fromages traités à la Pimaricine et placés en atmosphère d'ammoniac pendant 13 jours seulement subissent des variations de $\mathrm{pH}$; le $\mathrm{pH}$ évolue dans la croûte de 4,66 à 5,80 , de 4,60 à 5,20 dans la partie sous croûte et de 4,55 à 5,00 dans le centre (fig. 3 d).

Les variations brusques de $\mathrm{pH}$ observées dans les fromages avec Penicillium et notamment au niveau de la croûte coïncident avec l'implantation des moisissures.

b) Evolution des teneurs en calcium et phosphore

Les teneurs en calcium et phosphore de la croûte des fromages couverts de Penicililum et affinés en atmosphère non modifiée (fig. 4a5a) augmentent rapidement entre le $6^{\mathrm{e}}$ et $17^{\mathrm{e}}$ jour. Ainsi, pendant cette période des teneurs en calcium et phosphore passent respectivement de 4,10 à $24,50 \mathrm{~g} / \mathrm{kg}$ et de 3,50 à $17,70 \mathrm{~g} / \mathrm{kg}$. A partir du $6^{\mathrm{e}}$ jour les teneurs en ces éléments diminuent d'abord dans la partie sous croûte puis le centre. Les teneurs en minéraux de ces 2 parties s'équilibrent rers 1 e $17^{\mathrm{e}}$ jour à $1 \mathrm{~g} / \mathrm{kg}$ pour le calcium et $2 \mathrm{~g} / \mathrm{kg}$ pour le phosphore.

La présence d'ammoniac dans l'atmosphère d'affinage provoque une augmentation rapide des teneurs en calcium et phosphore dans la croûte et par voie de conséquence une diminution rapide de celles de la partie interne. Ainsi à 6 jours les teneurs en ces éléments, dans la croûte des fromages placés en atmosphère non modifiée, sont de 5,43 et $3,95 \mathrm{~g} / \mathrm{kg}$ alors que celles des fromages en atrnosphère ammoniacale sont respectivement de 12,75 et $7,95 \mathrm{~g} / \mathrm{kg}$. A 17 jours leurs teneurs sont pratiquement identiques dans les 2 types de fromage.

Les fromages traités à la pimaricine et placés en atmosphère non modifiée (fig. 4c-5c) ne sont le siège d'aucune migration minérale. Les teneurs en calcium et phosphore sont identiques dans les 3 parties.

Les fromages traités à la pimaricine et placés en atmosphère ammoniacale (fig. 4d-5d) subissent une migration minérale. Les teneurs en calcium et phosphore de la croûte passent respectivement de 4,10 à $9,82 \mathrm{~g} / \mathrm{kg}$ et 3,50 à $6,86 \mathrm{~g} / \mathrm{kg}$ à 13 jours. La présence d'ammoniac seul entraîne une migration minérale de l'intérieur vers l'extérieur du fromage.

\section{Influence de la teneur initiale en calcium et phosphore}

L'ultrafiltration au $\mathrm{pH}$ du lait conduit à l'obtention de fromages camembert plus minéralisés que les fromages issus de procédés tra- 


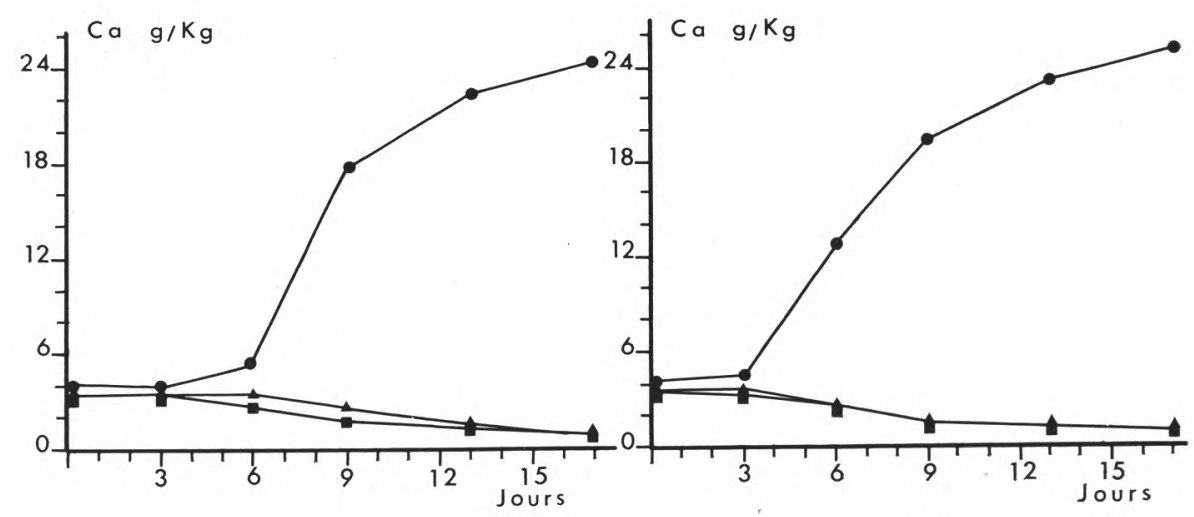

$4 a$

$4 \mathrm{~b}$

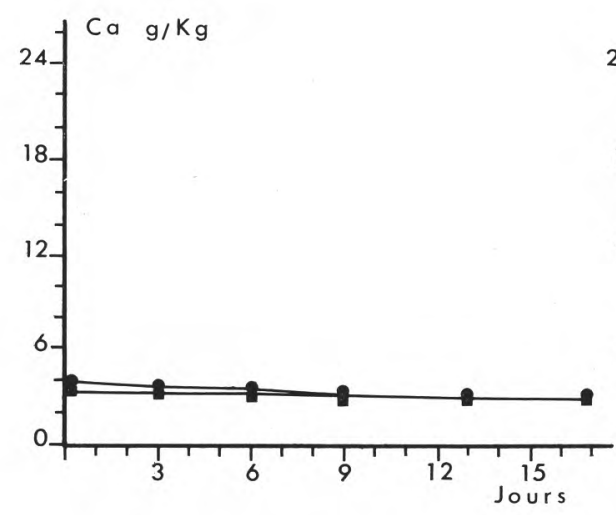

$4 \mathrm{c}$

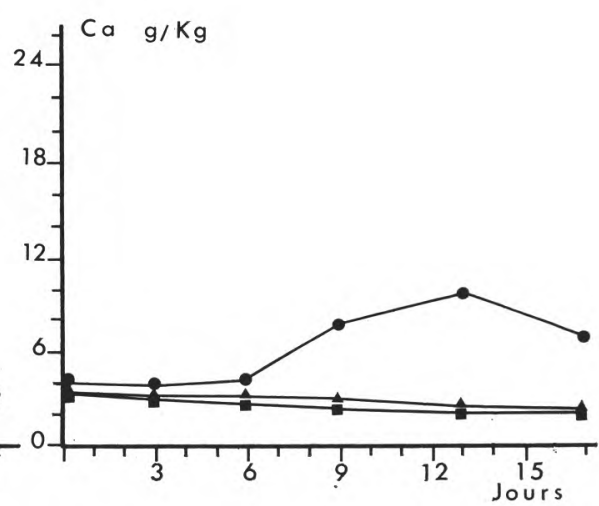

$4 d$

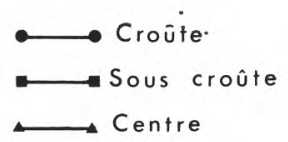

fig. 4

Evolution des teneurs en calcium au cours de l'affinage :

- fromages ensemencés avec $P$. caseicolum (4a - atmosphère non modifiée ; $4 \mathrm{~b}$ - atmosphère ammoniacale) ;

- fromages traités à la pimaricine $(4 \mathrm{c}$ - atmosphère non modifiée ; $4 \mathrm{~d}$. atmosphère ammoniacale).

Evolution of calcium contents during ripening :

- 4a - P. caseicolum - Normal conditions;

- $4 \mathrm{~b}$ - P. caseicolum - $\mathrm{NH}_{3}$ conditions;

- 4c - Pimaricin - Normal conditions;

- 4d - Pimaricin - $\mathrm{NH}_{3}$ conditions. 


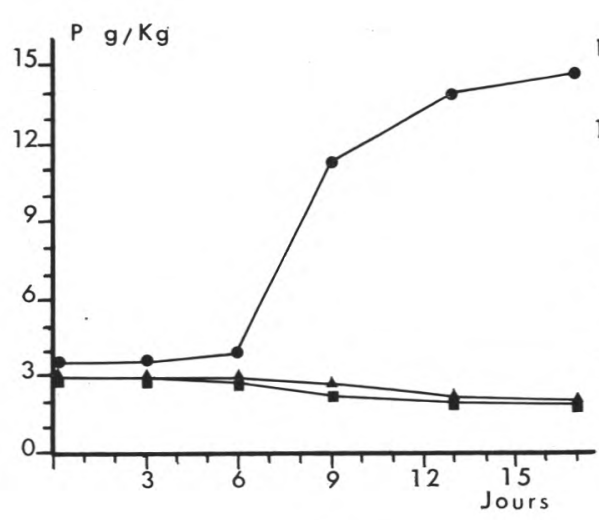

$5 a$

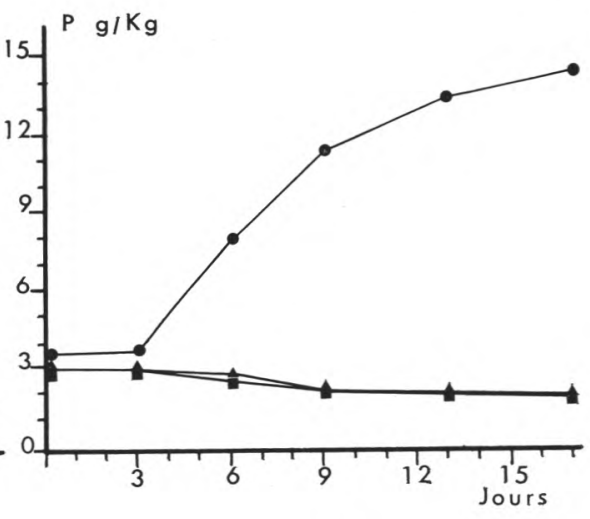

5 b

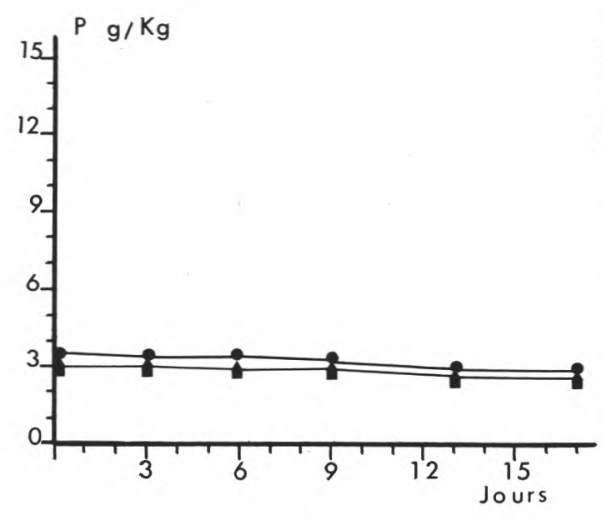

$5 \mathrm{c}$

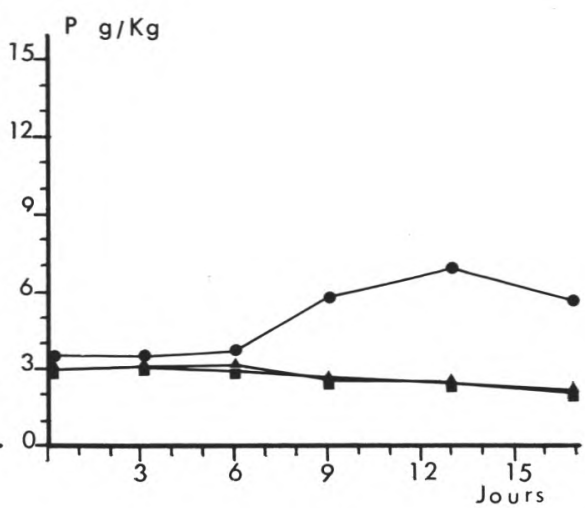

$5 d$

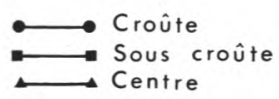

fig. 5

Evolution des teneurs en phosphore au cours de l'affinage :

- fromages ensemencés avec $P$. caseicolum (5a - atmosphère non modifiée ; $5 \mathrm{~b}$ - atmosphère ammoniacale ;

- fromages traités à la pimaricine $(5 c$ - atmosphère non modifiée ; $5 d$ atmosphère ammoniacale).

Evolution of phosphorus contents during ripening:

- 5a - P. caseicolum - Normal conditions;

- 5b - P. caseicolum - $\mathrm{NH}_{3}$ conditions;

- 5c - Pimaricin - Normal conditions;

- 5d - Pimaricin - $\mathrm{NH}_{3}$ conditions. 
ditionnels ce qui peut entraîner des défauts organoleptiques (Maubois, 1978). Néanmoins pour les besoins de notre étude, des fromages ont été préparés selon ce protocole dans le but d'élever les teneurs initiales en calcium et phosphates des caillés (tab. 1). La comparaison

\section{TABLEAU 1-TABLE 1}

Teneurs en calcium et phosphore $(\mathrm{g} / \mathrm{kg})$ des fromages obtenus traditionnellement (I) et par ultrafitration du lait (II)

Calcium and phosphorus amounts of cheeses:

I - conventional cheese;

II - cheese from ultrafiltrated milk

\begin{tabular}{|c|c|c|c|c|c|}
\hline & & \multicolumn{2}{|c|}{ I } & \multicolumn{2}{|c|}{ II } \\
\hline & & $\mathrm{Ca}$ & $\mathrm{P}$ & $\mathrm{Ca}$ & $\mathrm{P}$ \\
\hline $\begin{array}{c}1 \\
\text { jour }\end{array}$ & $\begin{array}{l}\text { Croûte } \\
\text { Sous croûte } \\
\text { Centre }\end{array}$ & $\begin{array}{l}3,87 \\
3,42 \\
2,95\end{array}$ & $\begin{array}{l}3,87 \\
3,19 \\
2,97\end{array}$ & $\begin{array}{l}5,40 \\
4,73 \\
4,55\end{array}$ & $\begin{array}{l}4,44 \\
3,64 \\
3,49\end{array}$ \\
\hline $\begin{array}{c}21 \\
\text { jours }\end{array}$ & $\begin{array}{l}\text { Croûte } \\
\text { Sous croûte } \\
\text { Centre }\end{array}$ & $\begin{array}{r}25,72 \\
1,10 \\
1,00\end{array}$ & $\begin{array}{r}14,45 \\
2,08 \\
2,34\end{array}$ & $\begin{array}{r}27,70 \\
1,70 \\
1,02\end{array}$ & $\begin{array}{r}14,62 \\
2,13 \\
1,98\end{array}$ \\
\hline
\end{tabular}

de la distribution de ces éléments minéraux dans les 2 types de fromage montre qu'après 21 jours d'affinage, le taux de calcium dans le centre est identique $(1,00 \mathrm{~g} / \mathrm{kg})$ alors qu'initialement la différence était de $1,50 \mathrm{~g} / \mathrm{kg}$. Par contre, le taux de phosphore dans le centre du fromage traditionnel affiné est d'environ $15 \%$ plus élevé alors qu'au départ il était de $17 \%$ plus faible. Le rapport massique $\mathrm{Ca} / \mathrm{P}$ qui au départ est de 1,00 dans le fromage traditionnel et de l'ordre de 1,27 dans le fromage obtenu par ultrafiltration est compris entre 0,40 et 0,50 dans le centre à 21 jours.

\section{Influence de la nature des sels}

Les teneurs en calcium et phosphore des fromages témoins à 14 jours sont respectivement de 1,12 et $1,82 \mathrm{~g} / \mathrm{kg}$ dans le centre et de 21,12 et $12,02 \mathrm{~g} / \mathrm{kg}$ dans la croûte (tab. 2).

Les fromages ayant reçu du chlorure de calcium ont des teneurs en calcium et phosphore de 3,05 à $1,75 \mathrm{~g} / \mathrm{kg}$ dans le centre et 27,39 et $11,50 \mathrm{~g} / \mathrm{kg}$ dans la croûte. Une augmentation de la teneur en calcium n'entraîne donc pas une migration plus importante du phosphore. 
TABLEAU 2 - TABLE 2

Evolution du $\mathrm{pH}$, des teneurs en calcium et phosphore $(\mathrm{g} / \mathrm{kg})$ dans les fromages au cours de l'affinage Evolution of $p H$, calcium and phosphorus contents in cheeses during ripening

\begin{tabular}{|c|c|c|c|c|c|c|}
\hline & \multicolumn{2}{|c|}{$\mathrm{pH}$} & \multicolumn{2}{|c|}{$\mathrm{Ca}$} & \multicolumn{2}{|c|}{$\mathrm{P}$} \\
\hline & $3 j$ & $14 \mathrm{j}$ & $3 \mathrm{j}$ & $14 \mathrm{j}$ & $3 \mathrm{j}$ & $14 \mathrm{j}$ \\
\hline $\begin{array}{l}\text { Témoin }(\mathrm{NaCl}) \\
\quad \text { - croûte } \\
\text { - partie sous croûte } \\
\text { - centre }\end{array}$ & $\begin{array}{l}4,67 \\
4,47 \\
4,47\end{array}$ & $\begin{array}{l}7,40 \\
5,26 \\
4,63\end{array}$ & $\begin{array}{l}3,09 \\
3,12 \\
2,73\end{array}$ & $\begin{array}{r}21,12 \\
0,85 \\
1,12\end{array}$ & $\begin{array}{l}3,24 \\
2,96 \\
2,89\end{array}$ & $\begin{array}{r}12,02 \\
1,90 \\
1,82\end{array}$ \\
\hline $\begin{aligned} \mathrm{CaCl}_{2} & (+\mathrm{NaCl}) \\
& \text { - croûte } \\
& \text { - partie sous croûte } \\
& \text { - centre }\end{aligned}$ & $\begin{array}{l}4,39 \\
4,30 \\
4,29\end{array}$ & $\begin{array}{l}7,25 \\
4,96 \\
4,29\end{array}$ & $\begin{array}{l}7,09 \\
6,24 \\
5,76\end{array}$ & $\begin{array}{r}27,39 \\
3,12 \\
3,05\end{array}$ & $\begin{array}{l}3,57 \\
2,73 \\
2,69\end{array}$ & $\begin{array}{r}11,50 \\
1,85 \\
1,75\end{array}$ \\
\hline $\begin{array}{l}\mathrm{Na}_{2} \mathrm{HPO}_{4}(+\mathrm{NaCl}) \\
\quad \text { - croûte } \\
\text { - partie sous croûte } \\
\text { - centre }\end{array}$ & $\begin{array}{l}5,03 \\
4,69 \\
4,54\end{array}$ & $\begin{array}{l}7,22 \\
6,54 \\
4,95\end{array}$ & $\begin{array}{l}6,69 \\
1,81 \\
2,17\end{array}$ & $\begin{array}{r}17,22 \\
1,60 \\
0,46\end{array}$ & $\begin{array}{l}8,12 \\
3,83 \\
3,53\end{array}$ & $\begin{array}{l}9,42 \\
4,64 \\
3,37\end{array}$ \\
\hline
\end{tabular}


Dans les fromages ayant reçu du phosphate disodique les quantités de calcium et phosphore à 14 jours sont respectivement de 0,46 et $3,37 \mathrm{~g} / \mathrm{kg}$ dans le centre et 17,22 et $9,42 \mathrm{~g} / \mathrm{kg}$ dans la croûte. L'addition de phosphate disodique entraîne une migration plus importante de calcium; ainsi dans le centre la teneur en calcium est passée de $1,12 \mathrm{~g} / \mathrm{kg}$ dans le témoin à $0,46 \mathrm{~g} / \mathrm{kg}$.

La présence de calcium ou de phosphate dans les sels de salage à sec permet de modifier considérablement le rapport $\mathrm{Ca} / \mathrm{P}$; dans le centre du témoin à 14 jours il est de 0,61 alors qu'il est respectivement de 1,75 et de 0,14 dans les fromages salés avec des sels contenant du chlorure de calcium et du phosphate disodique. Nous pouvons noter également que si l'addition de phosphate permet un épuisement plus important du calcium du centre, elle retarde toutefois la migration en surface.

\section{Evolution de l'extrait sec des fromages}

Les fromages traditionnels et MMV placés en atmosphère non modifiée (fig. 6a et 6b) montrent qu'il y a une augmentation de la teneur en extrait sec de 41 à $45-46 \%$ dans le centre. Dans la croûte, cette teneur évolue de 47 à $38 \%$ à 17 jours pour le traditionnel et à $32 \%$ pour le MMV .

Les fromages traités à la pimaricine et placés en atmosphère non modifiée ne présentent aucune évolution de leur extrait sec (fig. 6c).

En atmosphère d'ammoniac, les fromages traités à la pimaricine ont une teneur en extrait sec constante dans la croûte alors que celle du centre passe de 41 à $44,5 \%$ (fig. 6 d).

\section{DISCUSSION}

Au cours de l'affinage des fromages à pâte molle de type camembert, une quantité importante de calcium et des phosphates migrent de l'intérieur vers l'extérieur. Ces résultats confirment ceux de Metje et Fanni (1978) et ceux de Jakubowski et Reps (1966). Le calcium et le phosphate s'insolubilisent en surface sous forme de phosphate dicalcique et tricalcique. Le rapport massique $\mathrm{Ca} / \mathrm{P}$ de la croûte est de 1,87 ce qui est proche de celui du phosphate tricalcique qui est de 1,93. La précipitation de ce sel en surface a été signalée également par Swiateck et Jaworski (1959).

Cette migration phosphocalcique coïncide avec le développement de la moisissure comme l'ont indiqué Metje et Fanni, 1978. La croissance du Penicillium entraîne une alcalinisation de la surface 


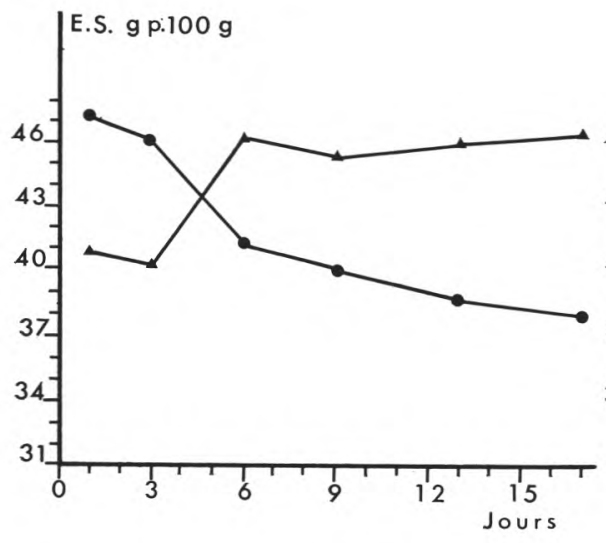

$6 a$

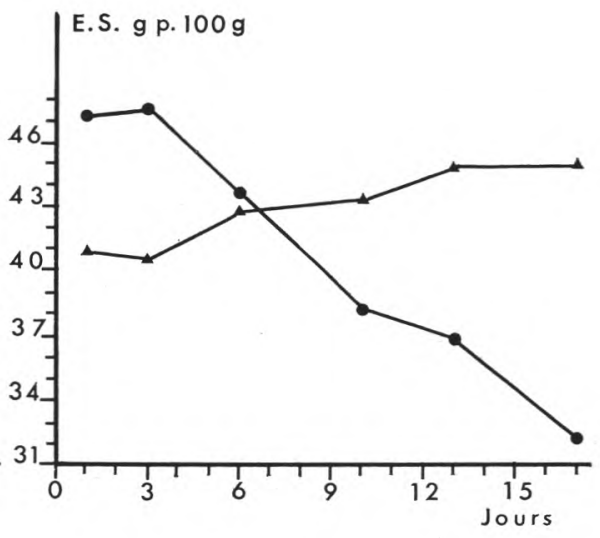

$6 \mathrm{~b}$

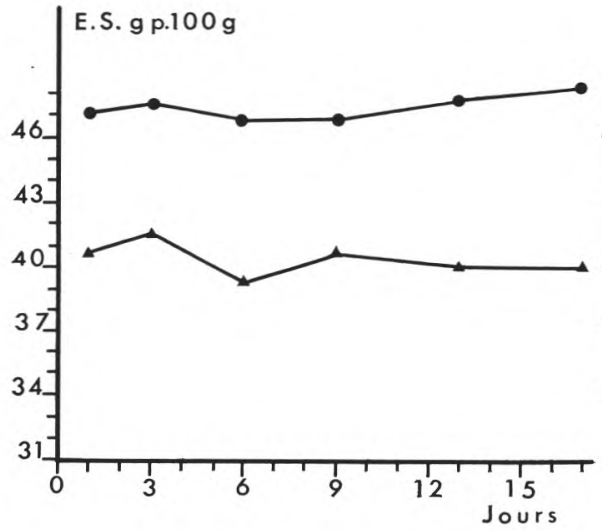

$6 c$

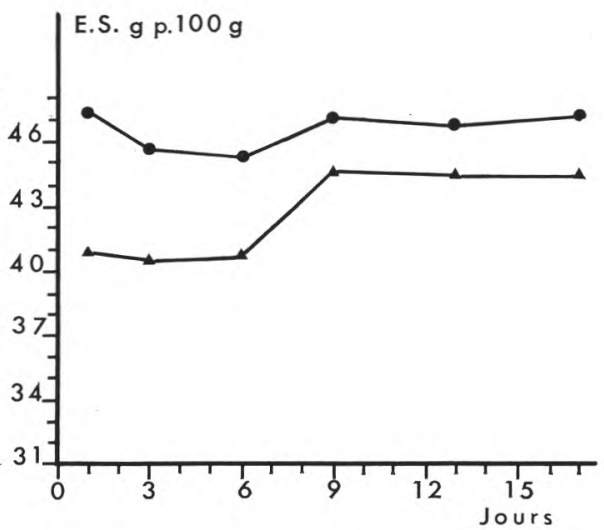

$6 d$

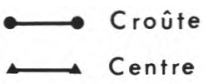

fig. 6

Evolution de l'extrait sec des fromages au cours de l'affinage :

- fromages ensemencés avec $P$. caseicolum et placés en atmosphère non modifiée (6a - traditionnel ; $6 \mathrm{~b}-\mathrm{MMV})$;

- fromages traditionnels traités à la pimaricine $(6 \mathrm{c}$ - atmosphère non modifiée ; $6 \mathrm{~d}$ - atmosphère ammoniacale) .

Evolution of dry matter during ripening:

- 6a - conventional cheese with P. caseicolum - Normal conditions;

- $6 \mathrm{~b}$ - cheese from ultrafiltred milk with P. caseicolum - Normal conditions;

- 6c - Pimaricin - Normal conditions;

- 6d Pimaricin - $\mathrm{NH}_{3}$ conditions. 
et crée ainsi un gradient de $\mathrm{pH}$ dans le fromage. Cette remontée du $\mathrm{pH}$ favorise la dissociation de la deuxième acidité des ions phosphates et entraîne la formation de phosphate dicalcique. Le calcium en neutralisant les deux premières acidités du phosphate exerce un appel d'électrons qui accroît alors la polarité de la troisième acidité et diminue son $\mathrm{pK}$ ce qui favorise la formation de phosphate tricalcique. La migration du calcium et des phosphates serait donc sous la dépendance du $\mathrm{pH}$, comme le montrent les résultats obtenus sur les fromages sans Penicillium placés en atmosphère ammoniacale et cec: malgré un gradient de $\mathrm{pH}$ nettement inférieur; il est, en effet, difficile de le recréer entièrement. Dans le cour du fromage, dont le pH est initialement de 4,6 , la totalité du calcium et du phosphate minéral est dissociée des protéines (Brulé et al., 1974), seul le phosphore organique est lié aux caséines. Ces ions $\mathrm{Ca}^{++}$ et $\mathrm{PO}_{4} \mathrm{H}_{2}-$ peuvent donc diffuser librement dans la phase aqueuse du fromage. La formation de phosphate de calcium, de très faible solubilité, favorisée par le pH plus élevé de la surface est responsable de l'immobilisation des ions calcium et phosphate dans la croûte et de l'épuisement progressif de ces ions dans l'intérieur de la pâte. La teneur en phosphore résiduel du centre du fromage correspond à la fraction organique. Celle-ci, est en plus faible quantité dans le cœur du fromage préparé à partir de lait ultrafiltré, ce qui s'explique par une teneur moindre en caséines puisque les protéines solubles dépourvues de phosphates sont retenues. La migration phosphocalcique dépend du rapport initial $\mathrm{Ca} / \mathrm{P}$ minéral et non pas de la concentration en chacun de ces ions; la migration est donc d'autant plus importante que la teneur initiale est importante. L'addition de chlorure de calcium au moment du salage ne permet pas une migration supérieure des phosphates. La teneur en phosphore du centre est de $1,82 \mathrm{~g} / \mathrm{kg}$ dans le fromage témoin et de $1,75 \mathrm{~g} / \mathrm{kg}$ dans le fromage salé en présence de chlorure de calcium. Ces résultats confirment que le phosphore résiduel est de nature organique. La stabilité des résidus phosphoséryles explique que les teneurs finales ne soient pas modifiées par l'apport de calcium.

L'addition de phosphate de sodium dans les sels de saumure, permet de réduire de moitié la teneur en calcium du centre après 21 jours d'affinage $(0,46 \mathrm{~g} / \mathrm{kg}$ contre $1,12 \mathrm{~g} / \mathrm{kg}$ dans le fromage témoin). L'ajout de chlorure de calcium et de phosphate de sodium montre que ce dernier est le facteur limitant de la migration minérale. Toutefois, malgré un excès de phosphate, la totalité du calcium ne migre pas ce qui peut s'expliquer par une remontée de $\mathrm{pH}$ plus rapide et en conséquence par une séquestration du calcium par les protéines ou peptides.

La migration des ions calcium et phosphate et la formation de phosphate tricalcique à la surface peuvent avoir des effets sur les caractéristiques de la pâte et de la croûte. Au cours de l'affinage, le calcium résiduel du fromage se refixe sur les caséines ou sur les phosphopeptides en raison de la remontée du $\mathrm{pH}$. Or nous savons que 
les propriétés rhéologiques de la pâte sont étroitement dépendantes du degré de minéralisation des protéines du réseau de paracaséine. La migration du calcium et la cristallisation de phosphate tricalcique peuvent s'accompagner d'une libération d'eau liée et favoriser des défauts de croûte.

L'évolution de l'extrait sec du centre et de la croûte du fromage au cours de l'affinage peut être comparée à celle des teneurs en phosphates et calcium, du $\mathrm{pH}$ puisqu'on note une variation importante entre 3 et $6 \mathrm{j}$; la migration d'eau du centre du fromage vers la croûte peut s'expliquer à la fois par le transfert des ions hydratés, par une augmentation de l'hydratation des protéines de surface consécutive à l'élévation du $\mathrm{pH}$ et par une absorption d'eau due au développement du Penicillium. Les fromages traités à la pimaricine et placés en atmosphère non modifiée qui ne subissent pas de variations de $\mathrm{pH}$, d'évolution minérale, ont un extrait sec stable ; par contre, en présence d'ammoniac, on note une variation de l'extrait sec du centre sans qu'il y en ait au niveau de la croûte ; ceci est probablement dû à une évaporation de surface plus importante en absence de Penicillium. Enfin, l'évolution de l'extrait sec minimise faiblement les variations des teneurs en phosphates et calcium des différentes parties du fromage au cours de l'affinage.

Sur un autre plan, il résulte de cette migration minérale vers la surface des fromages deux conséquences pratiques importantes. La première concerne l'apport minéral alimentaire (notamment calcique) par les fromages à pâte molle, selon que la croûte sera consommée ou non, puisqu'en fin d'affinage elle contient environ $80 \%$ du calcium et $55 \%$ du phosphore. La seconde a trait à l'analyse minérale elle-même de ce type de fromage. Les résultats obtenus varieront, en effet, baucoup selon que le prélèvement analysé contiendra ou non la croûte.

Enfin, il est permis de penser que des applications technologiques en matière de conduite de l'affinage peuvent découler de ces résultats. Il est en effet, probable que les mécanismes enzymatiques conduisant à l'évolution des textures et des flaveurs des pâtes fromagères soient fortement dépendants des condtions de pouvoir tampon et de force ionique existant dans la phase aqueuse interne du fromage. Or ces caractéristiques physico-chimiques peuvent être modifiées, dans une certaine mesure, par action sur la migration minérale périphérique. Par exemple, il peut être envisagé, pour agir sur cette migration, d'incorporer des phosphates dans les sels de saumurage ou de salage à sec, de modifier l'atmosphère des hâloirs (fromages à pâte molle) ou des caves d'affinage (autres fromages) ou encore d'utiliser des solutions salines spécifiques pour le lavage des pâtes fromagères (fromages à croûte lavée). C'est finalement tout un nouveau secteur d'investigations qui est ainsi ouvert aux technologues fromagers. 


\section{Remerciements}

Nous tenons à exprimer nos remerciements à $\mathrm{M}$. Maubois pour l'aide et les précieux conseils qu'il nous a apportés dans la rédaction de cette publication.

\section{Bibliographie}

Brûlé (G.), Maubois (J. L.) et Fauduant (J.) (1974). - Etude de la teneur en minéraux des produits obtenus lors de l'ultrafiltration du lait sur membrane. Le Lait, 54 (539-540), 600-615.

GUÉGUEN (L.) (1979). - Apports minéraux par le lait et les produits laitiers. Cahiers de nutrition et diététique, 14 (3), 215.

Guittonneau (G.) et ChevalieR (R.) (1935). - Sur les équilibres calco-phosphoriques réalisés dans les fromages. C.R. Académie des Sciences, 199, 801.

JaKUBOWSKI (J.) und RePS (A.) (1966). - Die durch das salzen hervorgerufene Migration von Wasser und Mineralstoffen im Käse. XVII ${ }^{\text {}}$ Congrès international de Laiterie (Section D : 2), 365-372.

Maubois (J. L.), Mocouot (G.) et Vassal (L.) (1969). - Procédé de traitement du lait et de ses sous-produits laitiers. Brevet français 2052121 .

Maubois (J. L.) (1978). - Application des techniques à membrane dans l'industrie fromagère. $\mathrm{XX}^{\mathrm{e}}$ Congrès international de Laiterie, Paris.

Metche (M.) et FanNi (J.) (1978). - Rôle de la flore fongique dans l'accumulation du calcium et du phosphore à la surface des fromages du type Camembert. Le Lait, 58 (77), 336-354.

RANDOIN (L.) et JouRdan (C.) (1951). - Détermination des teneurs en matière sèche, en calcium et en phosphore des principaux fromages français à pâte molle. Le Lait, 31 (303-304), 113-121.

SwIATEK (A.) und JaWORSKI (J.) (1959). - Histochemische Untersuchungen über die Verteilung von Mineralsalzen im Käse. XVe Congrès international de Laiterie, Londres (5a-40), 1509-1518. 